\title{
Thermal stability of Mo/Au bilayers for TES applications.
}

\author{
María Parra-Borderías ${ }^{1} \ddagger$, Iván Fernández-Martínez ${ }^{2,3}$, Lourdes \\ Fàbrega $^{4}$, Agustín Camón ${ }^{1}$, Oscar Gil ${ }^{4}$, Raquel \\ González-Arrabal $^{5}$, Javier Sesé ${ }^{6}$, José Luis Costa-Kramer ${ }^{2}$, \\ Bénédicte Warot-Fonrose ${ }^{7}$, Virginie Serin ${ }^{7}$ and Fernando \\ Briones $^{2}$ \\ ${ }^{1}$ Instituto de Ciencia de Materiales de Aragón and Departamento de Física de la \\ Materia Condensada, Universidad de Zaragoza - CSIC, Pedro Cerbuna 12, 50009 \\ Zaragoza, Spain \\ ${ }^{2}$ IMM-Instituto de Microelectrónica de Madrid, CNM-CSIC, Isaac Newton 8, PTM, \\ E-28760 Tres Cantos, Madrid, Spain \\ ${ }^{3}$ Instituto de Energía Solar, Avenida Complutense s/n, 28040 Madrid, Spain \\ ${ }^{4}$ Institut de Ciència de Materials de Barcelona, CSIC, Campus de la UAB, 08193 \\ Bellaterra, Spain \\ ${ }^{5}$ Instituto de Fusión Nuclear, Universidad Politécnica de Madrid, C/José Gutiérrez \\ Abascal 2, 28006 Madrid, Spain \\ ${ }^{6}$ Instituto de Nanociencia de Aragón, Universidad de Zaragoza, C/ Mariano Esquillor \\ s/n, 50018 Zaragoza, Spain \\ ${ }^{7}$ CEMES, 29 rue Jeanne Marvig BP 94347, 31055 Toulouse Cedex 4, France \\ E-mail: mariapb@unizar.es
}

\begin{abstract}
.
$\mathrm{Mo} / \mathrm{Au}$ bilayers are among the most suitable materials to be used as TransitionEdge Sensors (TES) in cryogenic microcalorimeters and bolometers, developed among other fields for space missions. For this purpose the thermal stability of TES at temperatures below $150^{\circ} \mathrm{C}$ is a critical issue.

We report on the dependence of functional properties (superconducting critical temperature, residual resistance and $\alpha$ ) as well as on microstructure, chemical composition and interface quality for optimized high quality $\mathrm{Mo} / \mathrm{Au}$ bilayers on annealing temperature and time. Data show that the functional properties of the bilayers remain stable at $T<150^{\circ} \mathrm{C}$, but changes in microstructure, interface quality and functional properties were observed for layers heated at $T \geqslant 200^{\circ} \mathrm{C}$. Microstructural and chemical composition data suggest that the measured changes in $R R R$ and $T_{C}$ at $T \geqslant 200^{\circ} \mathrm{C}$ are mainly due to an increase in the average Au grain size and to Au migration along the Mo grain boundaries at the $\mathrm{Au} / \mathrm{Mo}$ interface.

A way to stabilize the functional properties of the $\mathrm{Mo} / \mathrm{Au}$ bilayers against temperature enhancements is proposed.
\end{abstract}

\$ Present address: Centro de Astrobiología, INTA-CSIC, Carretera de Ajalvir km 4 28850, Torrejón de Ardoz, Madrid 
Thermal stability of Mo/Au bilayers for TES applications.

PACS numbers: 74.78.-w, 85.25.Oj, 74.45.+c, 74.62.Yb

Submitted to: Supercond. Sci. Technol. 


\section{Introduction}

High performance radiation detectors based on Transition-Edge Sensors (TES) are being developed for radiation detection in a wide range of frequencies, from millimetre waves $[1,2]$ to gamma-rays $[3,4]$. Among other fields such as particle physics, biochemistry and materials sciences, TES are considered indispensable in astronomy; nowadays, they are already used or proposed to be used in different telescopes on earth such as SCUBA2 [5] or APEX-SZ camera [6] and space missions, such as Micro-X sounding rocket [7], SAFARI instrument on board of SPICA [8], or XMS on former IXO mission and presently ATHENA $[9,10]$.

TES consist of a superconducting layer operated in the narrow temperature region between the normal and superconducting state. Proximity effect bilayers such as $\mathrm{Al} / \mathrm{Ag}[11], \mathrm{Ti} / \mathrm{Au}$ [12], $\mathrm{Mo} / \mathrm{Ag}$ [13], $\mathrm{Mo} / \mathrm{Cu}[14,15], \mathrm{Mo} / \mathrm{AuPd}$ [16] and $\mathrm{Mo} / \mathrm{Au}$ $[17,18,19,20]$, are preferred to single superconducting films [21]. Because of the proximity effect [22] in these bilayers, the superconducting transition temperature ( $T_{C, S}$ ) of the superconducting layer $(\mathrm{S})$ is reduced by the deposition of a normal metal layer (M) on top of it. When diminishing TES operation temperature down to 100 $\mathrm{mK}$ (the specified working temperature), the device sensitivity is observed to become up to two orders of magnitude higher than that of conventional detectors based on semiconductor thermistors. The TES sensitivity is mostly dictated by the sharpness of the superconducting transition, which turns out to ultimately depend on the S/M interface quality. Also, for device fabrication and operation the electrical resistivity is another point of concern $[23,24]$.

Because of the high melting point, high conductivity, good chemical stability and high hardness of Mo, the absence of intermetallic compounds and the lack of interdiffusion predicted for temperatures lower than $300^{\circ} \mathrm{C}$, Mo-based bilayers are foreseen to present a better response to ageing or temperature degradation than $\mathrm{Ti} / \mathrm{Au}$ or $\mathrm{Al} / \mathrm{Ag}$ bilayers $[17,25]$. Due to these features, to the excellent corrosion resistance of $\mathrm{Au}$ and their outstanding performance as radiation detectors, Mo/Au TES have been widely studied over the last decade [17, 18, 19, 20, 26].

Due to the possible temperature enhancements during launching, devices for space applications have to be stable. To ensure device integrity, thermal cycling test are required to discard outgassing, fracture of materials or assemblies due to dimensional tests or short circuiting of electrical wiring, among others. That means that TES have to preserve their functional properties in the temperature range specified in the test of flight $\left(T \leqslant 150^{\circ} \mathrm{C}\right)[27,28]$. Even when this condition is a must for space applications, to our knowledge the thermal stability of $\mathrm{Mo} / \mathrm{Au}$ bilayers has not been so far systematically studied. For that purpose, one of the key parameters to be considered is the steadiness of its interface. In principle, since Mo is a highly refractory material, annealing processes at temperatures below $600{ }^{\circ} \mathrm{C}$ are not expected to influence its microstructural properties [29]. This fact, together with the protection against oxidation or other impurities provided by the Au layer, allows foreseeing no change in its superconducting properties. 
However, the high mobility of gold atoms at temperatures as low as $200^{\circ} \mathrm{C}$ [30] could promote an increase in the $\mathrm{Au}$ grain size and/or Au migration in the interface region. These possible effects might induce variations in the electrical resistivity of the normal layer or deterioration of the $\mathrm{Mo} / \mathrm{Au}$ interface via stress generation and/or intermixing. In this paper, the thermal stability of high quality optimized $\mathrm{Mo} / \mathrm{Au}$ bilayers is studied. For this purpose, the critical temperature $\left(T_{C}\right)$ the superconducting transition width, the residual resistance $\left(R_{N}\right)$, the microstructural properties as well as the chemical composition of as-deposited and annealed bilayers at different temperatures and times are investigated.

\section{Experimental details}

Mo (50 nm) and Au (30 nm) layers were deposited at room temperature by RF and DC sputtering respectively, on $\mathrm{Si}$ (100) single crystal substrates covered by a $300 \mathrm{~nm} \mathrm{Si}_{3} \mathrm{~N}_{4}$ (deposited by low-pressure chemical vapour deposition, LPCVD). Prior to deposition the substrates were cleaned using a $\mathrm{KOH}$ solution $\left(15 \%\right.$ wt concentration) at $70^{\circ} \mathrm{C}$ for 4 minutes. The Ar working pressure was kept constant at $0.5 \mathrm{~Pa}$ during sputtering. Typical growth conditions for the Au layers were a DC power of $10 \mathrm{~W}$, giving rise to a deposition rate of $0.1 \mathrm{~nm} / \mathrm{s}$; while, for Mo layers, the RF power was fixed to keep voltage bias at a constant value of $230 \mathrm{~V}$, giving rise to a deposition rate of $0.1 \mathrm{~nm} / \mathrm{s}$. Such deposition conditions have been demonstrated to produce high quality, strain-free Mo layers [31, 32]. The temperature reached during deposition is not expected to be higher than $50^{\circ} \mathrm{C}$. Details on individual layer microstructure and bilayer functional properties, and their correlation to deposition conditions, are described elsewhere [31, 32].

Some of the bilayers were annealed at different temperatures $\left(100{ }^{\circ} \mathrm{C} \leqslant T \leqslant 300^{\circ} \mathrm{C}\right)$ for times between $30 \mathrm{~min}$ and $480 \mathrm{~min}$. in air atmosphere and at atmospheric pressure.

The superconducting critical temperature and the electrical resistance were measured on non-lithographed $\mathrm{Mo} / \mathrm{Au}$ bilayers of millimetric size were measured using the four-point resistance method through $25 \mu \mathrm{m}$ thick $\mathrm{Al}$ bonding wire. The use of non-lithographed samples was chosen to ensure that the changes observed with annealing temperature were due to variations on the Mo/Au bilayers and not to their manufacturing processes (lithography, contacting...). Measurements were carried out in a commercial Physical Property Measurement System (PPMS) with a ${ }^{3} \mathrm{He}$ option from Quantum Design by applying a constant current of $10 \mu \mathrm{A}$. Special care was taken to avoid remanent fields as much as possible by "quenching" the superconducting coil of the measurement system before every measurement.

The grain size of each layer and their interface quality were characterized by Transmission Electron Microscopy (TEM), using a F20 microscope fitted with a spherical aberration corrector. The interface chemical composition was analyzed by means of Electron Energy Loss Spectroscopy (EELS) in the same microscope using an Energy Filter GIF-Tridiem spectrometer. The spot diameter was $0.5 \mathrm{~nm}$ and the $\mathrm{L}_{2,3}(2520$ $\mathrm{eV})$ and $\mathrm{M}_{4,5}(2206 \mathrm{eV})$ edges were used for Mo and Au. The microstructure and 
residual stress were studied by X-ray diffraction (XRD) using a Bruker D8 Advance diffractometer with a GADDS 2-D detector.

In order to ensure that observed changes are due to thermal treatments and not to other factors such as ageing and/or irreproducibility in the measurement processes (due, for instance, to remnant fields or thermal coupling), samples prior to and after annealing were measured together with an as-deposited (control sample). In all the studied cases control samples properties remain invariable between different cryogenic runs.

\section{Results and discussion}

The temperature-induced changes in the residual resistance ratio $(R R R=$ $\left.R(300 K) / R_{N}\right)$ and in $T_{C}$ for bilayers annealed for $30 \mathrm{~min}$ at temperatures in the range from 100 to $300{ }^{\circ} \mathrm{C}$ are shown in figure 1 . Errors in $R R R$ are due to the measurement sensitivity, while $T_{C}$ errors account for possible variations in remanent fields (typically less than 2 Gauss after "quenching" the superconducting coil of the measurement system) or sample thermalization. $T_{C}$ error is obtained from the changes in $T_{C}$ measured for the control sample. These results evidence that annealings at $\mathrm{T} \leqslant 150^{\circ} \mathrm{C}$ do not lead to significant $R R R$ changes. However, $R R R$ slightly increases for samples annealed at $T \geqslant 200^{\circ} \mathrm{C}$.

As for the critical temperature, the bilayer annealed at $T \leqslant 100{ }^{\circ} \mathrm{C}$ exhibits the same $T_{C}$ value than the as-deposited. The $T_{C}$ of the bilayer annealed at $T=150{ }^{\circ} \mathrm{C}$ is slightly smaller by $4 \pm 1 \mathrm{mK}$ than that of the as-deposited sample. Nevertheless, the change is too small to significantly affect the TES final performance. For bilayers annealed at $T \geqslant 200^{\circ} \mathrm{C}, T_{C}$ slightly decreases by $\sim 10 \pm 1 \mathrm{mK}$.

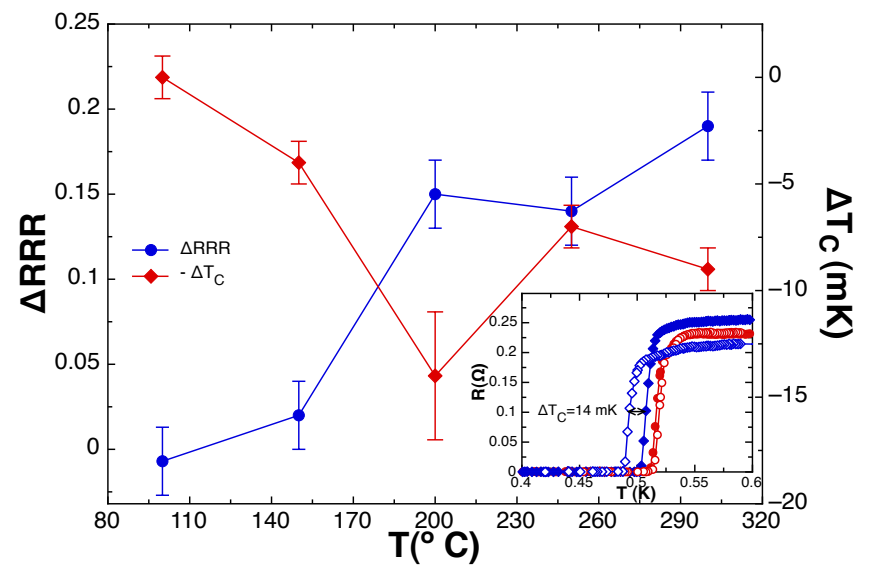

Figure 1: Variation in $R R R$ and $T_{C}$ as a function of the annealing temperature $\left(\Delta R R R=R R R_{\text {after annealing }}-R R R_{\text {prior to annealing }}\right.$ and $\Delta T_{C}=T_{C, \text { after annealing }}-$ $T_{C}$, prior to annealing). All samples were heated for 30 minutes. Inset displays $\mathrm{R}(\mathrm{T})$ measurement of a sample prior $(\bullet)$ and after $(\diamond)$ being heated at $200{ }^{\circ} \mathrm{C}$ for 30 minutes together with its control sample $(\bullet$ and $\circ)$.

We can therefore conclude that, within the error bars, both $R R R$ and $T_{C}$ display 
the same behaviour, with a step change when the annealing temperature increases from 150 to $200^{\circ} \mathrm{C}$.

As shown in the inset in figure 1, the sharpness of the superconducting transition (transition width $\sim 20 \mathrm{mK}$ ) is very much similar for as-deposited and all annealed samples. In this work we are dealing with non-lithographed samples, thus the $\operatorname{logarithmic}$ sensitivity value $(\alpha=d \log R / d \log T)$ of these bilayers is not expected to provide relevant information on the quality or sensitivity of the final sensor. Thus, a detailed determination of $\alpha$, that would require much more data points, was beyond the scope of the present study. From our $R(T)$ measurements $\alpha$ can be roughly estimated as $\sim 400$, which is an order of magnitude smaller than those usually reported in literature for lithographed samples of similar bilayers [33, 34, 35]. Within the obtained error bars, no change of $\alpha$ is observed in the measured temperature range, although results do not completely rule out small changes in $\alpha$.

The dependence of $T_{C}$ and $R R R$ on annealing time for samples heated at $T=200^{\circ} \mathrm{C}$ is shown in figure 2. No further time dependence of these values, or $\alpha$ is observed when samples are heated longer than $480 \mathrm{~min}$. This is a very relevant finding, as it indicates that one procedure to stabilize the bilayers against temperature enhancements would be to heat them for at least $480 \mathrm{~min}$. For annealing temperatures where no change in $T_{C}$, $R R R$ and $\alpha$ was observed $\left(T \leqslant 100^{\circ} \mathrm{C}\right)$, these functional properties remain invariable even when heated for longer annealing times i.e. 120 min. (not shown).

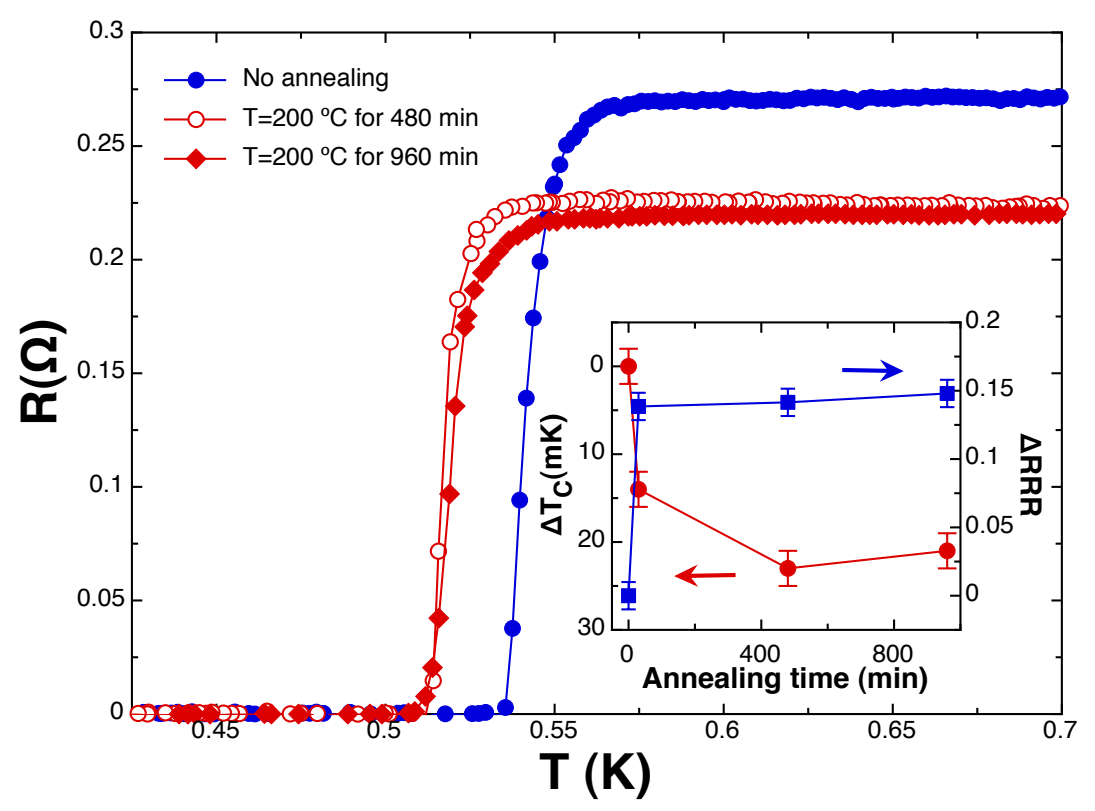

Figure 2: $R(T)$ measurement of a sample before and after being heated at $200{ }^{\circ} \mathrm{C}$ for 480 min and $960 \mathrm{~min}$. Inset displays variation in $R R R$ and $T_{C}$ as a function of the annealing time for samples heated at $200{ }^{\circ} \mathrm{C}$.

In order to account for experimental observations, microstructural studies were carried out for Mo and Au layers and their interface. Moreover, the chemical composition 
of the $\mathrm{Mo} / \mathrm{Au}$ interface and $\mathrm{Mo} / \mathrm{Si}_{3} \mathrm{~N}_{4}$ (substrate) was also characterized.

XRD measurements (not shown) evidence that annealing induces no significant modifications in the stress states of Mo and Au layers in the studied temperature range. The grain size in as-deposited and annealed bilayers was characterised by means of cross-sectional TEM images. TEM images shown in figure 3 reveal that the Mo average column width, estimated as displayed in figure 3, does not change by annealing, whereas the average Au grain size increases from $19 \mathrm{~nm}$ for the as-deposited bilayer to $25 \mathrm{~nm}$ for that annealed at $300^{\circ} \mathrm{C}$ for $30 \mathrm{~min}$. To ensure a sufficiently large statistics, these values were obtained averaging results over more than 30 grains, obtained from different TEM images.

As it is well known, the resistance of a metal has two components: the electron scattering with phonons and that with lattice imperfections. The first one, typically proportional to temperature, mainly depends on the phonon concentration; the second one, known as residual resistance, is the one which dominates the low temperature behavior. Since no variation of the resistance temperature dependent part with annealing is observed after eliminating geometrical factors, the measured $R R R$ changes might be necessarily related to variations in the residual resistance of the Au layer.

Thus, the measured enhancement in the Au average grain size will lead to a reduction of the grain boundary scattering contribution to the residual resistivity. This term can be expressed in granular films like our Au layers, according to MayadasShatzkes[36], as:

$$
\rho_{M}^{\text {bulk }}=\rho_{M}^{\text {film }} 3\left[\frac{1}{3}-\frac{a}{2}+a^{2}-a^{3} \ln \left(1+\frac{1}{a}\right)\right]
$$

Where $a$ is defined as $\frac{R}{1-R} \frac{\ell_{0}}{D}$, being $D$ the average grain size, $R$ a factor corresponding to the fraction of electrons specularly reflected at the boundary, $\rho_{M}^{\text {bulk }}$ is the residual resistivity of a bulk sample, $\rho_{M}^{f i l m}$ is the residual resistivity of the film and $\ell_{0}$ the electron mean free path of the metal. Assuming that $\rho_{M}^{\text {bulk }}=0.022 \cdot 10^{-8} \Omega \mathrm{m}$ [37], $\ell_{0}=40 \mathrm{~nm}[38]$, and $\rho_{M}^{\text {film }}$ the residual resistance measured for our samples, a $\mathrm{R}$ value $\sim 0.43$ (similar to other data found for (111) evaporated Au layers[30]) reproduces the changes in $R R R$ after annealing. Thus the observed enhancement in the average Au grain size quantitatively accounts for the measured resistance change.

Regarding $T_{C}$, even though the electrical resistivity can affect the bilayer $T_{C}$ for thick enough layers [39], the measured residual resistance variation is too low to justify the annealing-induced $T_{C}$ changes. Therefore, $T_{C}$ variations might be mostly related to alterations in the interface quality [39], and thus, in the coupling between Mo and Au layers.

In order to study the interface chemical sharpness EELS measurements were carried out. EELS compositional profiles for an as-deposited and an annealed bilayer at $300{ }^{\circ} \mathrm{C}$ for 30 minutes are depicted in figure 4 . It is observed that annealing nearly doubles the extension of the region in which the chemical composition is different from pure $\mathrm{Au}$ and Mo (from $1.1 \pm 0.3 \mathrm{~nm}$ for the as-deposited to $2.1 \pm 0.3 \mathrm{~nm}$ for the annealed samples). Such 


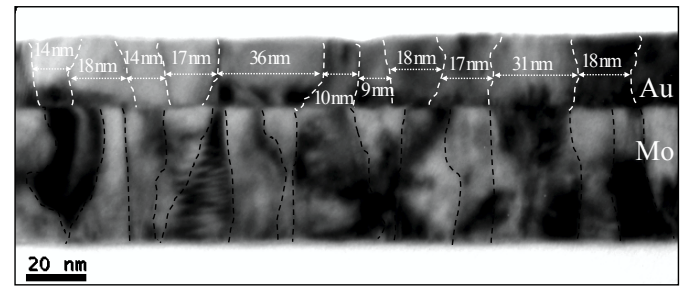

(a)

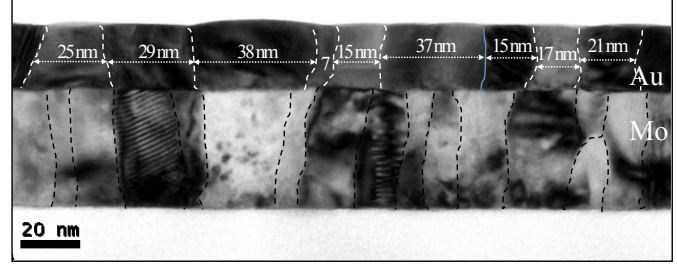

(b)

Figure 3: (a) Cross-section TEM image of a 50/30 nm Mo/Au bilayer before being heated at $300{ }^{\circ} \mathrm{C}$ for 30 minutes. (b) Cross-section TEM image of a 50/30 nm Mo/Au bilayer after being heated at $300{ }^{\circ} \mathrm{C}$ for 30 minutes.

behaviour has been systematically observed for all profiles in all analyzed samples, while the interface roughness, determined as the average distance between peaks and valleys in different TEM images, slightly alters, as it might be expected considering that, because of its high refractory character, Mo grains and microstructure will hardly be modified by the temperatures used in the studied heat treatments. These data can be explained by considering that Au migrates somehow in between Mo columns without significantly modify the interface roughness. Such a modification in the chemical composition at the interface might affect the coupling between Mo and Au layers, providing a plausible explanation for the observed $T_{C}$ changes for samples heated at $T \geqslant 200{ }^{\circ} \mathrm{C}$. This change in the interface quality might also affect $\alpha$; in fact is has been shown above that small $\alpha$ changes can not be fully ruled out.

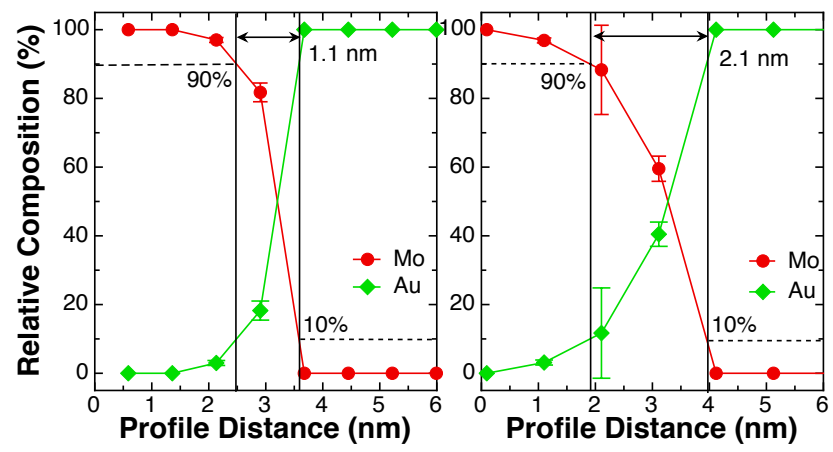

Figure 4: Quantification EELS profiles along the Mo/Au interface for an as-deposited 50/30 $\mathrm{nm} \mathrm{Mo/Au} \mathrm{bilayer} \mathrm{(Left)} \mathrm{and} \mathrm{for} \mathrm{a} 50 / 30 \mathrm{~nm} \mathrm{Mo/Au}$ bilayer after heating the sample to 300 ${ }^{\circ} \mathrm{C}$ for 30 minutes (Right). 
Finally, to discard a possible influence of the substrate in the measured $T_{C}$ changes, EELS compositional profiles of the Mo-substrate interface were also performed in an as-deposited and an annealed bilayer at $300{ }^{\circ} \mathrm{C}$ for 30 minutes. As displayed in figure 5 , no change in the chemical composition at the $\mathrm{Mo}^{-} \mathrm{Si}_{3} \mathrm{~N}_{4}$ interface is observed within the error margins. This fact, together with the absence of additional superconducting transitions discards the formation of high $T_{C}$ superconducting compounds of $\mathrm{N}$ or $\mathrm{Si}$ and Mo.

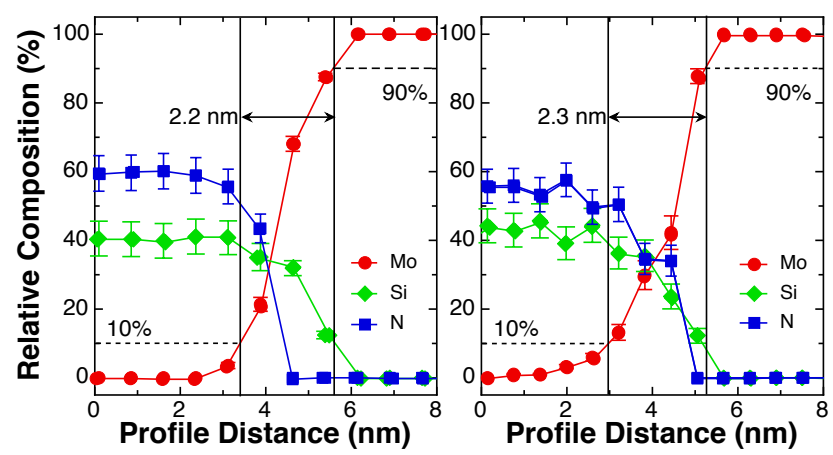

Figure 5: Quantification EELS profiles along the $\mathrm{Mo} / \mathrm{Si}_{3} \mathrm{~N}_{4}$ interface for an as-deposited 50/30 $\mathrm{nm} \mathrm{Mo/Au} \mathrm{bilayer} \mathrm{(Left)} \mathrm{and} \mathrm{for} \mathrm{a} 50 / 30 \mathrm{~nm} \mathrm{Mo/Au} \mathrm{bilayer} \mathrm{after} \mathrm{heating} \mathrm{the} \mathrm{sample} \mathrm{at} 300$ ${ }^{\circ} \mathrm{C}$ for 30 minutes (Right).

\section{Conclusions}

We have shown that functional properties of $\mathrm{Mo} / \mathrm{Au}$ bilayers are stable in the temperature range specified in the test of flight $\left(T \leqslant 150{ }^{\circ} \mathrm{C}\right)$, which makes them competitive candidates for future space missions. We present a complete study on how $T_{C}, R R R$ and $\alpha$ for optimized sputtered Mo/Au bilayers deposited on $\mathrm{Si}_{3} \mathrm{~N}_{4}$ are modified by annealing them at temperatures above or equal to $200^{\circ} \mathrm{C}$, well below the predicted limit for $\mathrm{Mo} / \mathrm{Au}$ interdiffusion. The measured $R R R$ changes are related to $R_{N}$ variations that are associated to the increment in the Au average grain size, while the main reason for the observed $T_{C}$ changes might be the atomic migration of Au inbetween Mo grain boundaries. No change in $\alpha$ is found within the error limits, regardless of annealing time and temperature.

Despite these changes, we find out that, if required by any future application, bilayers can be make stable against further heating in the studied temperature and time ranges by heating the bilayers at $T \geqslant 200^{\circ} \mathrm{C}$ for at least $480 \mathrm{~min}$. after their deposition.

\section{Acknowledgments}

This work has been carried out with financial support from the Spanish MICINN (projects ESP2006-13608-C02, AYA2008-00591/ESP, MAT2008-01077/NAN and MAT2009-13977-C03). The authors acknowledge financial support from the 
European Union 6th Framework program (Integrated Infrastructure Initiative- 026019 ESTEEM). MPB and OG would like to thank Spanish Education Ministry and MICINN for a FPU grant and a FPI grant and for the Juan de la Cierva financial support. We thank J.Albiol for helpful discussions.

\section{References}

[1] Lee A T, Richards P L, Nam S W, Cabrera B and Irwin K D 1996 Appl. Phys. Lett. 69 1801-1803

[2] Myers M J, Holzapfel W, Lee A T, O'Brient R, Richards P L, Tran H T, Ade P, Engargiola G, Smith A and Spieler H 2005 Appl. Phys. Lett. 86114103

[3] Cunningham M, Ullom J N, Miyazaki T, Labov S, Clarke J, Lanting T M, Lee A T, Richards P L and Yoon J 2002 Appl. Phys. Lett. 81 159-161

[4] Chow D, Lindeman M, Cunningham M, Frank M, Jr T B and Labov S 2000 J. Low Temp. Phys. 444

[5] Woodcraft A L et al. 2007 Rev. Sci. Instrum. 78024502

[6] Mehl J et al. 2008 J. Low Temp. Phys. 151 697-702

[7] Wikus $\mathrm{P}$ et al. 2009 AIP Conf. Proc. 1185 434-437

[8] Swinyard B and Nakagawa T 2009 Exp. Astron. 23(1) 193-219

[9] den Herder J W et al. 2010 SPIE Proc. Ser. 7732 77321H

[10] Kelley R et al. 2009 AIP Conf. Proc. 1185

[11] Wollman D A, Irwin K, Hilton G C, Dulcie L, Newbury D and Martinis J 1997 J. Microsc. 188 196-223

[12] Bruijn M P, Tiest W M B, Hoevers H F C, Krouwer E, van de Kuur J, Ridder M L, Moktadir Z, Wiegerink R, van Gelder D and Elwenspoek M 2003 Nucl. Instrum. Meth. A 513 143-146

[13] Monticone E, Rajteri M, Portesi C, Gandini C, Bodoardo S and Picotto G 2002 Physica C 372 440-443

[14] Hilton G C, Beall J A, Deiker S, Vale L R, Doriese W B, Beyer J, Ullom J N, Reintsema C D, Xu $\mathrm{Y}$ and Irwin K D 2004 Nucl. Instrum. Meth. A $\mathbf{5 2 0}$ 435-438

[15] Goldie D J, Audley M D, Glowacka D M, Tsaneva V N and Withington S 2008 J. Appl. Phys. 103 084509

[16] Boucher R, May T, Wagner T, Zakosarenko V, Anders S and Mayer H G 2006 Supercond. Sci. Technol. 19 138-142

[17] Finkbeiner F M, Chen T C, Aslam S, Figueroa-Feliciano E, Kelley R L, Li M, Mott D, Stahle C and Stahle C 1999 IEEE T. Appl. Supercon. 9 2940-2942

[18] Tralshawala N, Brekosky R, Li M, Figueroa-Feliciano E, Finkbeiner F, Lindeman M, Stahle C and Stahle C 2001 IEEE T. Appl. Supercon. $11755-758$

[19] Chervenak J A et al. 2004 Nucl. Instrum. Meth. A 520 460-462

[20] Bandler S R et al. 2008 J. Low Temp. Phys. 151 400-405

[21] Irwin K D and Hilton G C 2005 Cryogenic Particle Detection vol 99 (Springer-Verlag) pp 63-149

[22] Werthamer N 1963 Phys. Rev. 132 2440-2445

[23] Hilton G, Martinis J, Irwin K, Bergren N, Wollman D, Huber M, Deiker S and Nam S 2001 IEEE T. Appl. Supercon. $11739-742$

[24] Irwin K D, Hilton G C, Wollman D A and Martinis J M 1998 J. Appl. Phys. 83 3978-3985

[25] Massalski T B (ed) 1990 Binary Alloy Phase Diagrams 2nd ed vol 1 (ASM International)

[26] Glowacka D M, Goldie D J and Withington S 2010 ISST Proc. 276-278

[27] Document Standardization Division 2006 MIL-STD-883- Test Methods and Procedures for Microelectronics, Test method 1010 Defense Logistics Agency Columbus, E.E.U.U.

[28] European Cooperation for Space Standarization 2008 ECSS-Q-ST-70-04C-Thermal testing for the evaluation of space materials, processes, mechanical parts and assemblies ESA Requirements and Standards Division Noordwijk, The Netherlands 
[29] Oikawa H and Nakajima Y 1977 J. Vac. Sci. Technol. 14 1153-1156

[30] Sambles J R, Elsom K C and Jarvis D J 1982 Philos. T. R. Soc. S-A 304 365-396

[31] Fàbrega L, Fernández-Martínez I, Parra-Borderías M, Gil O, Camón A, González-Arrabal R, Sesé J, Santiso J, Costa-Krämer J L and Briones F 2009 IEEE T. Appl. Supercon. 19 3779-3785

[32] Fàbrega L et al. 2009 IEEE T. Appl. Supercon. 19 460-464

[33] Luukanen A, Sipilä H, Kinnunen K, Nuottajärvi A and Pekola J 2000 Physica B 284-288 2133 2134

[34] Tralshawala N, Aslam S, Brekosky R P, Chen T, Figueroa-Feliciano E, Finkbeiner F M, Li M, Mott D, Stahle C and Stahle C 2000 Nucl. Instrum. Meth. A 444 188-191

[35] Chen T C, Finkbeiner F M, Bier A and DiCamillo B 1999 Supercond. Sci. Technol. 12840

[36] Mayadas A F, Shatzkes M and Janak J F 1969 Appl. Phys. Lett. 14 345-347

[37] Lide D 1996 Handbook of Chemistry and Physics 75th ed (New York: CRC Press)

[38] Fawcett E and Griffiths D 1962 J. Phys. Chem. Solids $231631-1635$

[39] Martinis J M, Hilton G C, Irwin K D and Wollman D A 2000 Nucl. Instrum. Meth. A 444 23-27 